\title{
Millimeter wave spectra of ethyl isocyanate and searches for it in Orion KL and Sagittarius B2^
}

\author{
L. Kolesniková ${ }^{1}$, E. R. Alonso ${ }^{1}$, B. Tercero ${ }^{2}$, J. Cernicharo ${ }^{3}$, and J. L. Alonso ${ }^{1}$ \\ ${ }^{1}$ Grupo de Espectroscopia Molecular (GEM), Edificio Quifima, Área de Química-Física, Laboratorios de Espectroscopia y \\ Bioespectroscopia, Parque Científico UVa, Unidad Asociada CSIC, Universidad de Valladolid, 47011 Valladolid, Spain \\ e-mail: lucie.kolesnikova@uva.es \\ 2 Observatorio Astronómico Nacional (OAN-IGN), Calle Alfonso XII, 3, 28014 Madrid, Spain \\ 3 Instituto de Ciencia de Materiales de Madrid, CSIC, C/ Sor Juana Inés de la Cruz 3, 28049 Cantoblanco, Spain \\ Received 12 April 2018 / Accepted 23 May 2018
}

\begin{abstract}
Context. Relatively high abundances of methyl isocyanate $\left(\mathrm{CH}_{3} \mathrm{NCO}\right)$, a methyl derivative of isocyanic acid (HNCO), found in the Orion KL and Sgr B2 molecular clouds suggest that its ethyl derivative, ethyl isocyanate $\left(\mathrm{CH}_{3} \mathrm{CH}_{2} \mathrm{NCO}\right)$, may also be present. Aims. The aim of this work is to provide accurate experimental frequencies of ethyl isocyanate in its ground and excited vibrational states in the millimeter wave region to support searches for it in the interstellar medium.

Methods. The rotational spectrum of ethyl isocyanate was recorded at room temperature from 80 to $340 \mathrm{GHz}$ using the millimeter wave spectrometer in Valladolid. Assigned rotational transitions were analyzed using the $S$-reduced semirigid-rotor Hamiltonian. Results. More than 1100 distinct frequency lines were analyzed for the ground vibrational state of the cis conformer as well as for three vibrational satellites corresponding to successive excitation of the lowest-frequency $\mathrm{C}-\mathrm{N}$ torsional mode. Newly determined rotational and centrifugal distortion constants were used for searches of spectral features of ethyl isocyanate in Orion KL and Sgr B2 clouds. Upper limits to $\mathrm{CH}_{3} \mathrm{CH}_{2} \mathrm{NCO}$ in these high-mass star-forming regions were obtained.
\end{abstract}

Key words. astrochemistry - ISM: molecules - astronomical databases: miscellaneous - ISM: individual objects: Orion KL - ISM: individual objects: Sgr B2

\section{Introduction}

Two isocyanate- (-NCO) containing compounds have been detected to date in the interstellar medium (ISM). Isocyanic acid, HNCO, was one of the first interstellar molecules (Snyder \& Buhl 1972) and now it is known to be present with high abundances in a variety of Galactic sources as well as in external galaxies (see Brünken et al. 2010, and the references therein). The search for methyl derivative of the isocyanic acid, methyl isocyanate $\left(\mathrm{CH}_{3} \mathrm{NCO}\right)$, has been motivated by its identification on the frozen surface of comet $67 \mathrm{P} /$ ChuryumovGerasimenko (Goesmann et al. 2015). It was followed by its detection in the high-mass star-forming regions Orion KL (Cernicharo et al. 2016) and Sagittarius (Sgr) B2 (Halfen et al. 2015; Cernicharo et al. 2016; Belloche et al. 2017) and in low-mass protostar IRAS 16293-2422 (Ligterink et al. 2017; Martín-Doménech et al. 2017) with relatively high abundances. For example, the abundance of this species in Orion KL is only a factor of ten below those of $\mathrm{HNCO}$ and $\mathrm{CH}_{3} \mathrm{CN}$ (Cernicharo et al. 2016). Taking into account the significant abundances of other ethyl derivatives of very abundant species in Orion $\mathrm{KL}$ and $\mathrm{Sgr} \mathrm{B} 2\left(\mathrm{CH}_{3} \mathrm{CH}_{2} \mathrm{CN}, \mathrm{CH}_{3} \mathrm{CH}_{2} \mathrm{OH}\right.$, and $\mathrm{CH}_{3} \mathrm{CH}_{2} \mathrm{OCOH}$ ), the ethyl derivative of isocyanic acid, ethyl isocyanate $\left(\mathrm{CH}_{3} \mathrm{CH}_{2} \mathrm{NCO}\right)$, could be thus considered as a good candidate for detection in these hot cores.

\footnotetext{
* Tables 4 and 5 are only available in electronic form at the CDS via anonymous ftp to cdsarc.u-strasbg.fr (130.79.128.5) or via http://cdsweb.u-strasbg.fr/cgi-bin/qcat?J/A+A/616/ A173
}

Owing to the high leOwing to the high level of line blending and line confusion in the millimeter wave spectra of high-mass star-forming regions (see, e.g., Tercero et al. 2010; Belloche et al. 2013), the identification of a new molecule in these sources has to be ensured by the detection of several features that follow the characteristic spectral pattern of the molecule (see, e.g., Tercero et al. 2015). For this reason, high-quality laboratory spectroscopy is an essential step before any astrophysical search. To date, $\mathrm{CH}_{3} \mathrm{CH}_{2} \mathrm{NCO}$ has been studied in the gas phase using conventional Stark-modulation spectroscopy (Sakaizumi et al. 1976) and a waveguide (Kasten et al. 1983) and molecular beam (Heineking et al. 1994) Fourier transform microwave spectroscopy. Nevertheless, these spectroscopic data do not exceed $35.5 \mathrm{GHz}$. Therefore, to avoid common problems resulting from extrapolation, new laboratory measurements up to $340 \mathrm{GHz}$ have been performed. Directly measured transition frequencies together with a new set of spectroscopic constants have been used to search for $\mathrm{CH}_{3} \mathrm{CH}_{2} \mathrm{NCO}$ in the ISM. Results are discussed in the following sections.

\section{Experimental details}

The sample of liquid ethyl isocyanate was obtained commercially and was used without any further purification. The rotational spectra were recorded employing the millimeter wave spectrometer, which is based on cascaded multiplication of the primary synthesizer frequency (up to $20 \mathrm{GHz}$ ) by a set of active and passive multipliers. A detailed description of the spectrometer can be found in Daly et al. (2014). In this case, the millimeter wave region from 80 to $340 \mathrm{GHz}$ was covered by amplifier- 


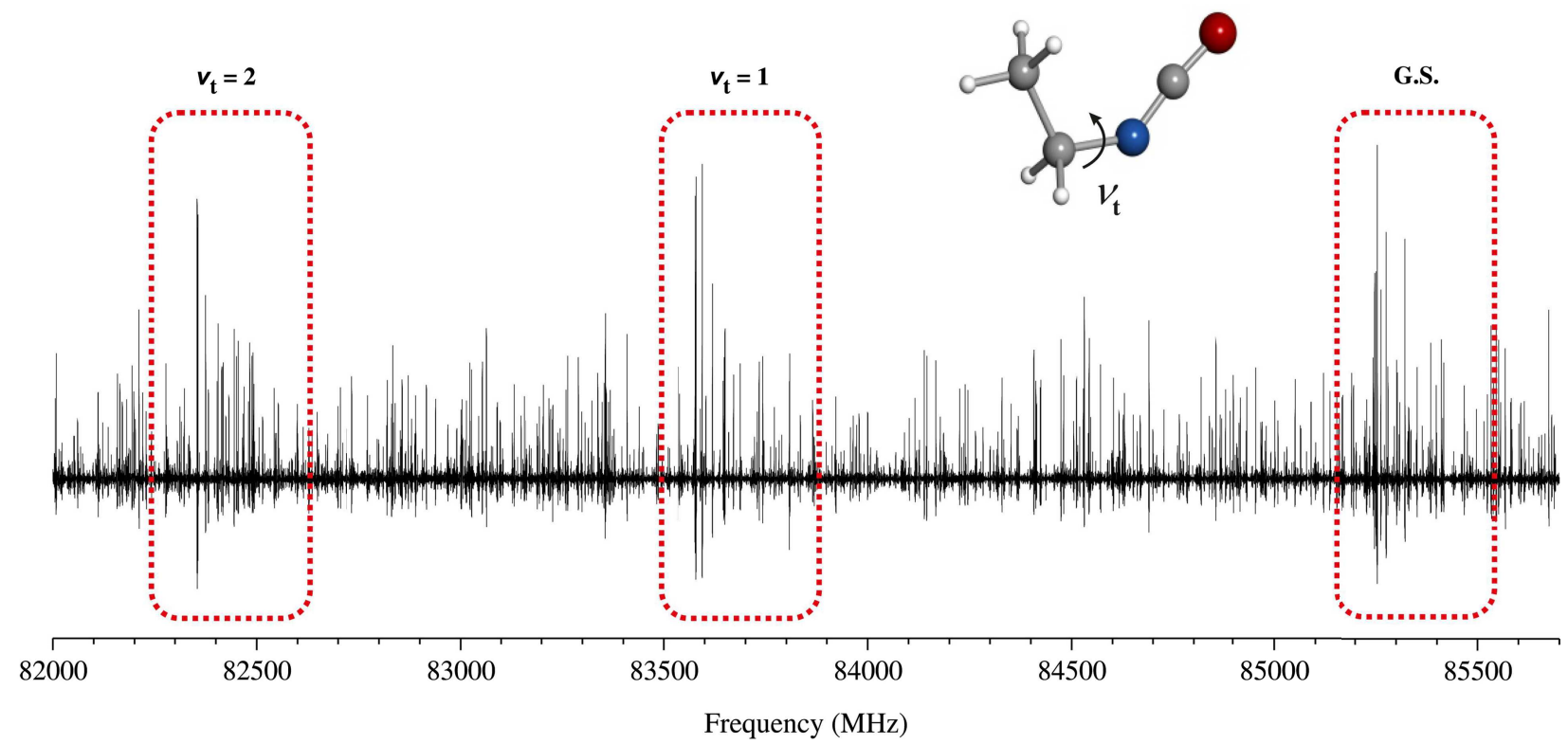

Fig. 1. Representative $3.7 \mathrm{GHz}$ segment of room-temperature millimeter wave spectrum of ethyl isocyanate illustrating its rather complex nature. Groups of $a$-type $R$-branch transitions $(J=15 \leftarrow 14)$ for the ground state (G.S.) and two successive excitations of the C-N torsional mode $\left(v_{t}=1,2\right)$ are marked.

multiplier chains WR10.0, WR6.5, and WR9.0 (VDI, Inc) in combination with an additional frequency doubler and tripler (WR4.3, WR2.8, VDI, Inc.). The synthesizer frequency was modulated at $f=10.2 \mathrm{kHz}$ with modulation depth ranging from 30 to $40 \mathrm{kHz}$. A detection system composed of either Schottky diodes or a broadband quasioptical detector (VDI, Inc) was completed by a $2 f$ demodulation using a phase sensitive lock-in amplifier with time constant set to $30 \mathrm{~ms}$. The second derivative shape of the lines was fitted to the Gaussian profile function, and the uncertainty of the line center frequency was estimated to be better than $50 \mathrm{kHz}$ for isolated well-developed lines. All the spectra were recorded in $1 \mathrm{GHz}$ sections in both directions (a single acquisition cycle) and averaged. The frequency step size between adjacent data points of 30 and $50 \mathrm{kHz}$ has been applied below and above $170 \mathrm{GHz}$, respectively, leading to an integration time of 19 and $32 \mathrm{~min}$ for a $1 \mathrm{GHz}$ piece of the spectra. During the measurements, the sample of ethyl isocyanate was kept at room temperature and pressure between 10 and $20 \mu$ bar.

\section{Rotational spectra and analysis}

The room-temperature millimeter wave rotational spectrum of ethyl isocyanate in Fig. 1 presents an exceptionally high line density. Its analysis was started using predictions from the spectroscopic constants obtained in the previous microwave works (Heineking et al. 1994; Kasten et al. 1983; Sakaizumi et al. 1976) for the stable cis configuration. It is characterized by a relatively large dipole moment along the $a$ inertial axis $\left(\left|\mu_{a}\right|=2.81\right.$ $\mathrm{D}$ and $\left|\mu_{b}\right|=0.03 \mathrm{D}$ (Sakaizumi et al. 1976), $C_{\mathrm{s}}$ symmetry, and dihedral angle $\tau(\mathrm{C}-\mathrm{C}-\mathrm{N}=\mathrm{C})=0^{\circ}$ (see Fig. 1). The identification of $K_{a}=0$ and lower-frequency $K_{a}=1$ transitions, originating from $J_{0} J$ and $J_{1 J}$ energy levels, was relatively straightforward and their assignment and analysis could be easily expanded up to $340 \mathrm{GHz}\left(J^{\prime \prime}=64\right)$ with the help of the Loomis-Wood-type plot technique from the AABS package (Kisiel et al. 2005) and SPFIT/SPCAT program suite (Pickett 1991). The upper-frequency $K_{a}=1$ transitions ( $J_{1} J-1$ levels), which became degenerate with $K_{a}=2$ transitions ( $J_{2} J-1$ levels) for $J^{\prime \prime}>45$, were subsequently localized, however, their analysis quickly ran into problems.
As shown in Fig. 2, significant departures from the predicted positions were observed at high $J^{\prime \prime}$ even though it was clear from the Loomis-Wood-type plots that the assignments were correct. Similar situations also occurred for higher $K_{a}$ transitions and with increasing value of $K_{a}$, the limit of $J$ up to which the rotational transitions were amenable to the simple semirigid-rotor Hamiltonian analysis, was decreasing (see Fig. 2). Only those transitions that supported the semirigid-rotor treatment were retained in the analysis. These transitions were finally combined with hyperfine-free transitions $\left(5-24 \mathrm{GHz}, J^{\prime \prime}=0-7, K_{a}^{\prime \prime}=0-3\right.$ ) from Heineking et al. (1994) and globally fitted using Watson's $S$-reduced Hamiltonian in $I^{r}$ representation. The adjusted rotational and centrifugal distortion constants are given in Table 1. The list of experimental frequencies is provided in the Table 4. Finally, the deviation trends observed in the Loomis-Wood type plots, such as those in Fig. 2, were advantageously followed to measure the frequencies of more than 200 transitions that could not be encompassed in the semirigid rotor fit. These transitions are collected in the Table 5 .

Apart from the ground vibrational state, three vibrational satellites could be observed in the spectrum. These satellites were assigned to three successive excitations $\left(v_{t}=1,2,3\right)$ of $\mathrm{C}-\mathrm{N}$ torsional mode $v_{t}$, which is expected to be the lowest frequency mode (Durig et al. 2010; Sullivan et al. 1987; Sakaizumi et al. 1976). A torsional frequency of about $40 \mathrm{~cm}^{-1}$ can be roughly estimated from the relative intensity measurements. Considerable perturbations were also observed in the rotational spectra of these excited states and, as in case of the ground state, only the less perturbed transitions were used to obtain the spectroscopic constants in Table 1. The inertia defect values for the ground state as well as for the $v_{t}=1,2,3$ excited states can be found in the same table. The increase of their negative values with the vibrational quantum number corroborate the assignment of the satellite lines as belonging to an out-of-plane vibration. 


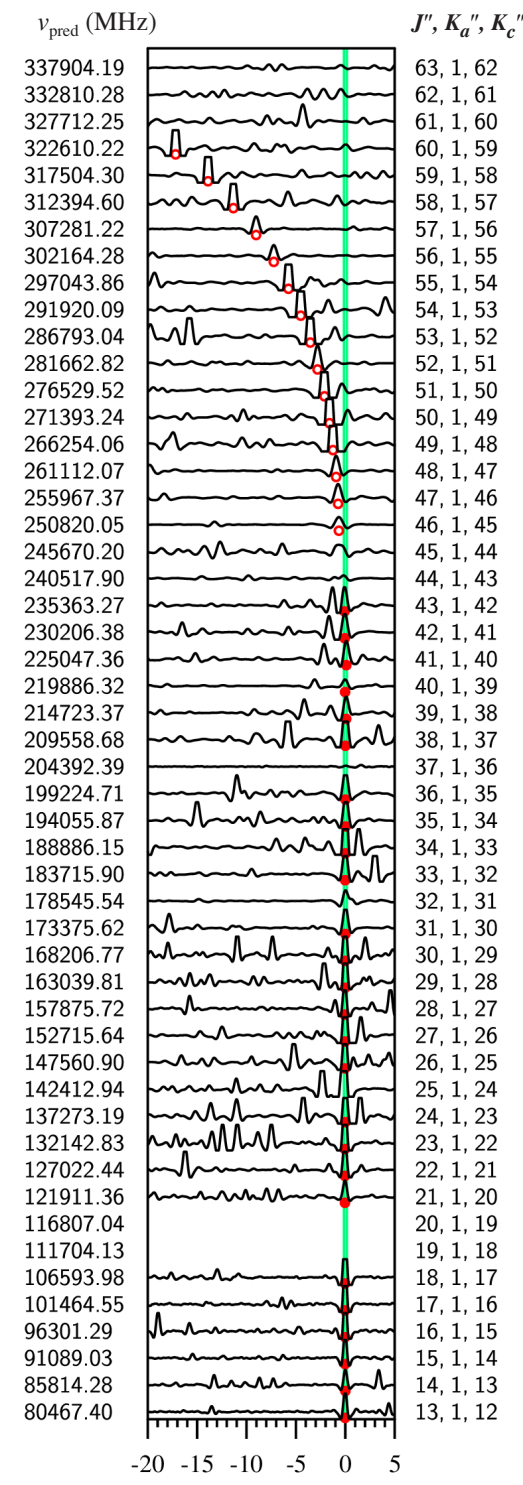

$\Delta v(\mathrm{MHz})$

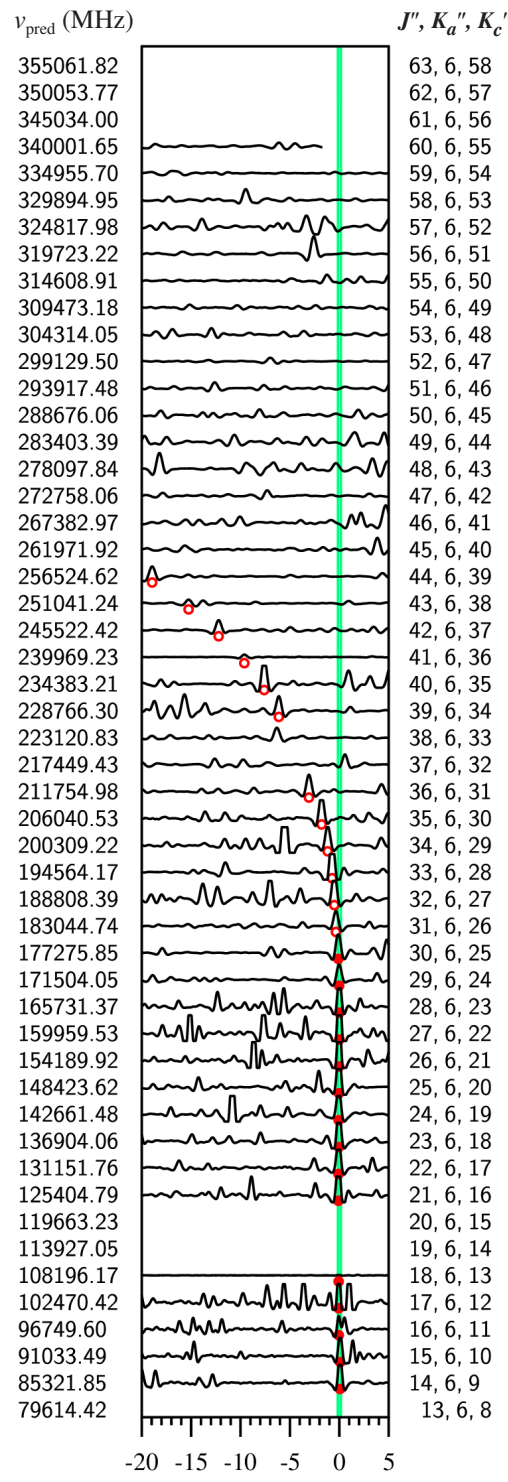

$\Delta v(\mathrm{MHz})$

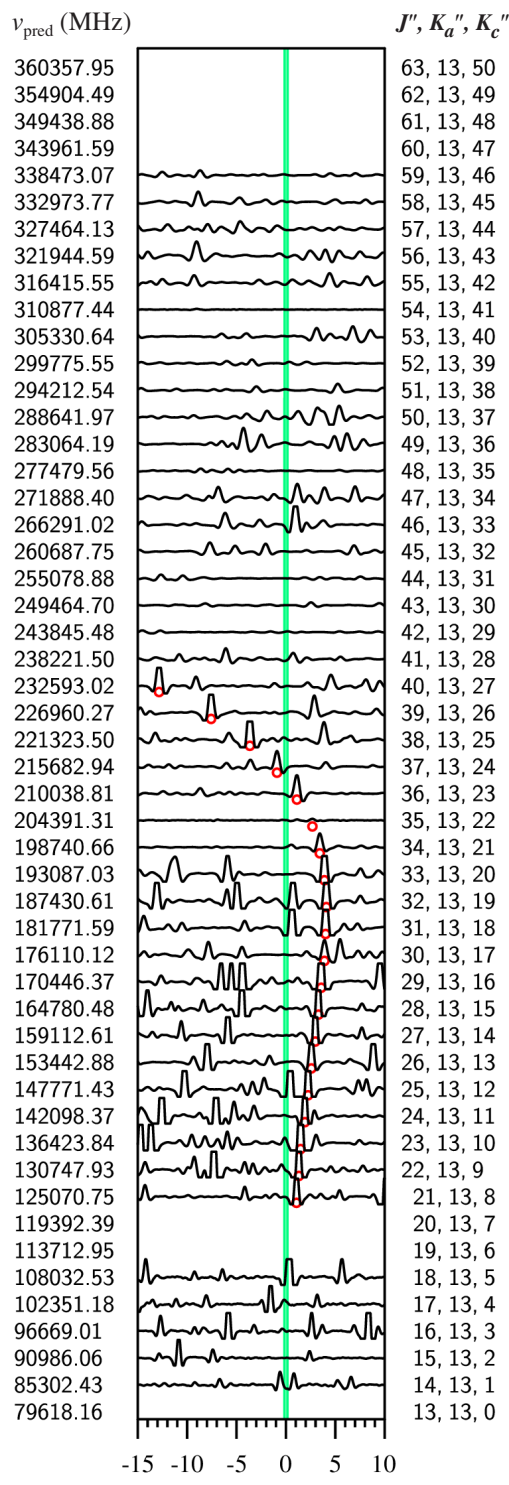

$\Delta v(\mathrm{MHz})$

Fig. 2. Sample Loomis-Wood-type plots of the rotational spectrum of ethyl isocyanate for $K_{a}=1, K_{a}=6$, and $K_{a}=13$ line sequences in the ground vibrational state where sections of the experimental spectrum are plotted around the predicted positions (green vertical line) using the spectroscopic constants from Table 1. Predicted frequency is given on the left side of each plot while the lower state rotational quantum numbers $J^{\prime \prime}, K_{a}^{\prime \prime}$, and $K_{c}^{\prime \prime}$ are shown on the right side. Frequency distance from the predicted positions is given by $\Delta v$. Red filled circles mark the transition lines that are included in the present semirigid rotor Hamiltonian analysis (Table 4). Red opened circles identify perturbed transitions not included in the fit (Table 5).

\section{Searching for ethyl isocyanate in space}

Motivated by the high abundances of methyl isocyanate $\left(\mathrm{CH}_{3} \mathrm{NCO}\right)$ found in comet $67 \mathrm{P} / \mathrm{Churyumov-Gerasimenko}$ (Goesmann et al. 2015), in the high-mass star-forming regions Orion KL (Cernicharo et al. 2016) and Sagittarius (Sgr) B2 (Halfen et al. 2015; Cernicharo et al. 2016), and in low-mass protostars (Ligterink et al. 2017; Martín-Doménech et al. 2017), we conducted the search for its ethyl derivative. As we pointed out previously, the perturbations found in the laboratory spectra of ethyl isocyanate prevent accurate predictions of many $a$-type transitions at frequencies above $150 \mathrm{GHz}$. Only a limited number of $K_{a}=0,1,2,3$ transitions could be assigned with an uncertainty below $0.1 \mathrm{MHz}$. Thus, it is not possible to search for a straightened pattern of lines at these frequencies. Therefore, in this work we focused the search for $\mathrm{CH}_{3} \mathrm{CH}_{2} \mathrm{NCO}$ in the $3 \mathrm{~mm}$ spectral window (80$\left.115 \mathrm{GHz}, \mathrm{HPBW} \sim 30-21^{\prime \prime}\right)$ using the public data of Orion KL and Sgr B2 collected with the Institut de Radioastronomie Millimétrique (IRAM) $30 \mathrm{~m}$ telescope (Tercero et al. 2010; Belloche et al. 2013).

We did not detect ethyl isocyanate above the detection limit of these data. In Table 2, upper limits to the $\mathrm{CH}_{3} \mathrm{CH}_{2} \mathrm{NCO}$ column density are provided and Fig. 3 shows the non-detection of this species in the considered data set. To derive these upper limits we used the MADEX code (Cernicharo 2012) introducing the new measured laboratory transitions of this work and using the Local Thermodynamic Equilibrium (LTE) approximation. The physical parameters adopted for the model were those derived by Cernicharo et al. (2016) for $\mathrm{CH}_{3} \mathrm{NCO}$ in these sources. Cor- 
Table 1. Spectroscopic constants in the ground state and $v_{t}=1,2,3$ of ethyl isocyanate ( $S$-reduction, $I^{r}$-representation).

\begin{tabular}{lrrrr}
\hline \hline Constant / Unit & G.S. & $v_{t}=1$ & $v_{t}=2$ & $v_{t}=3$ \\
\hline$A / \mathrm{MHz}$ & $14114.8138(46)^{a}$ & $14656.90(14)$ & $15218.37(21)$ & $15924.57(54)$ \\
$B / \mathrm{MHz}$ & $3067.18293(26)$ & $2982.5564(18)$ & $2923.1693(24)$ & $2861.2419(60)$ \\
$C / \mathrm{MHz}$ & $2604.87552(23)$ & $2578.2917(13)$ & $2556.6437(16)$ & $2533.1756(31)$ \\
$D_{J} / \mathrm{kHz}$ & $4.60971(69)$ & $5.9662(12)$ & $6.8962(18)$ & $8.6185(58)$ \\
$D_{J K} / \mathrm{kHz}$ & $-39.562(12)$ & $-70.314(23)$ & $-96.605(19)$ & $-147.498(69)$ \\
$D_{K} / \mathrm{kHz}$ & $353.2(21)$ & $609.0(95)$ & $1008(18)$ & $1478(98)$ \\
$d_{1} / \mathrm{kHz}$ & $-1.49562(30)$ & $-2.07372(84)$ & $-2.4051(14)$ & $-3.0355(47)$ \\
$d_{2} / \mathrm{kHz}$ & $-0.05569(29)$ & $-0.13799(44)$ & $-0.14165(78)$ & $-0.1375(33)$ \\
$H_{J} / \mathrm{Hz}$ & $-0.10972(46)$ & $-0.18033(41)$ & $-0.22578(85)$ & $-0.5468(42)$ \\
$H_{J K} / \mathrm{Hz}$ & $-1.115(28)$ & $1.098(14)$ & $1.677(11)$ & $5.034(67)$ \\
$H_{K J} / \mathrm{Hz}$ & $4.503(38)$ & $-23.92(13)$ & $-55.34(15)$ & $-163.09(54)$ \\
$h_{1} / \mathrm{Hz}$ & $-0.06043(27)$ & $-0.10570(37)$ & $-0.11326(78)$ & $-0.2659(39)$ \\
$h_{2} / \mathrm{Hz}$ & $-0.00606(19)$ & $-0.02032(25)$ & $-0.00896(65)$ & $-0.0131(27)$ \\
$h_{3} / \mathrm{Hz}$ & $0.000442(76)$ & $-0.00185(17)$ & $-0.00183(33)$ & $-0.00143(98)$ \\
$L_{J} / \mathrm{mHz}$ & $-0.000360(21)$ & $\ldots$ & $\ldots$ & $\ldots$ \\
$L_{J J K} / \mathrm{mHz}$ & $-0.882(19)$ & $\ldots$ & $\ldots$ & $\ldots$ \\
$\Delta / \mathrm{uA}{ }^{2}$ & $-6.561901(25)$ & $-7.91241(36)$ & $-8.42301(49)$ & $-8.8609(12)$ \\
$N_{\text {lines }} b$ & 331 & 246 & 206 & 114 \\
$\sigma_{\text {fit }}{ }^{c} / \mathrm{MHz}$ & 0.053 & 0.072 & 0.064 & 0.069 \\
\hline
\end{tabular}

Notes. ${ }^{(a)}$ The numbers in parentheses are $1 \sigma$ uncertainties (67\% confidence level) in units of the last decimal digit. ${ }^{(b)}$ Number of distinct frequency lines in the fit. ${ }^{(c)}$ Root mean square deviation of the fit.

Table 2. Physical parameters of the considered cloud cores.

\begin{tabular}{lccc}
\hline \hline & Orion KL & \multicolumn{2}{c}{ Sgr B2 } \\
\cline { 3 - 4 } & & Comp. 1 & Comp. 2 \\
\hline$v_{\text {LSR }}\left(\mathrm{km} \mathrm{s}^{-1}\right)$ & 7 & 63 & 73 \\
$\Delta v_{\text {FWHM }}\left(\mathrm{km} \mathrm{s}^{-1}\right)$ & 5 & 7 & 7 \\
$d_{\text {sou }}^{a}\left(^{\prime \prime}\right)$ & 8 & 2.4 & 1.9 \\
offset $^{b}\left({ }^{\prime \prime}\right)$ & 5 & 0 & 0 \\
$T_{\text {rot }}(\mathrm{K})$ & $150 \pm 50$ & $200 \pm 60$ & $200 \pm 60$ \\
$N\left(\mathrm{CH}_{3} \mathrm{NCO}^{c}\left(\mathrm{~cm}^{-2}\right)\right.$ & $(5 \pm 2) \times 10^{15}$ & $(1.5 \pm 0.5) \times 10^{17}$ & $(9 \pm 3) \times 10^{16}$ \\
$N\left(\mathrm{CH}_{3} \mathrm{CH}_{2} \mathrm{NCO}\right)\left(\mathrm{cm}^{-2}\right)$ & $\leq(5 \pm 2) \times 10^{14}$ & $\leq(1.5 \pm 0.5) \times 10^{16}$ & $\leq(9 \pm 3) \times 10^{15}$ \\
\hline
\end{tabular}

Notes. ${ }^{(a)}$ Source size diameter. ${ }^{(b)}$ With respect to the pointing position of the data (see Tercero et al. 2010; Cernicharo et al. 2016). ${ }^{(c)}$ Cernicharo et al. (2016).

rections for beam dilution were applied to each line depending on their frequency. Interestingly, we derived a column density ratio $N\left(\mathrm{CH}_{3} \mathrm{NCO}\right) / N\left(\mathrm{CH}_{3} \mathrm{CH}_{2} \mathrm{NCO}\right) \geq 10$ in both sources.

Table 3 shows the relative column densities of Methyl(Met)/Ethyl(Et) of other species in Orion KL and Sgr B2. Firstly, we noted that, in addition to different chemical paths, the large disagreement of the Met/Et ratios in Orion is related to the detection of the ethyl species in spatially different components. Whereas $\mathrm{CH}_{2} \mathrm{CH}_{3} \mathrm{CN}$ is mainly detected in the "classical" hot core of Orion, $\mathrm{CH}_{3} \mathrm{CH}_{2} \mathrm{OH}$ peaks towards a position $5^{\prime \prime}$ south-west of the hot core (a similar location to the emission peak of $\mathrm{CH}_{3} \mathrm{NCO}$ ) and $\mathrm{CH}_{3} \mathrm{CH}_{2} \mathrm{OCOH}$ emission arises from the compact ridge component, which differs both physically and chemically from the hot core (see, e.g., Cernicharo et al. 2016 and references therein for a detailed description of the Orion KL components). Nevertheless, it is worth noting that the lower limit ratio derived for Met-NCO/Et-NCO is consistent with the Met/Et ratios of O-bearing species in both sources.

Belloche et al. (2009) derived column density ratios of related species of ethyl formate $\left(\mathrm{C}_{2} \mathrm{H}_{5} \mathrm{OCHO}\right)$ and $n$-propyl cyanide $\left(\mathrm{C}_{3} \mathrm{H}_{7} \mathrm{CN}\right)$ in Sgr B2. Their chemical modeling for these results in Sgr B2(N) (63-64 $\mathrm{km} \mathrm{s}^{-1}$ component) suggests that the most likely formation route for ethyl cyanide is a sequential construction of this species from their constituent functional groups on the grain surfaces. Of course, this model explains the depletion of $\mathrm{CH}_{3} \mathrm{CN}$ in hot cores in which large abundances of ethyl cyanide are found and it is consistent with the Met-CN/Et-CN ratios found in Orion KL and Sgr B2 (see Table 3). Alternatively, Belloche et al. (2009) proposed that ethyl formate is primarily formed on the grains by adding $\mathrm{HCO}$ or $\mathrm{CH}_{3}$ to functional-group radicals derived from methyl formate $\left(\mathrm{CH}_{3} \mathrm{OCOH}\right)$ and ethanol $\left(\mathrm{CH}_{3} \mathrm{CH}_{2} \mathrm{OH}\right)$. Moreover, in the chemistry of cold $\mathrm{H}$ addition reactions with $\mathrm{CO}$ in interstellar grains, methanol $\left(\mathrm{CH}_{3} \mathrm{OH}\right)$ and ethanol $\left(\mathrm{CH}_{3} \mathrm{CH}_{2} \mathrm{OH}\right)$ are formed by means of different precursors: $\mathrm{H}_{2} \mathrm{CO}$ and $\mathrm{CH}_{3} \mathrm{CHO}$, respectively (see, e.g., Herbst \& van Dishoeck 2009). These formation paths point out that there is not a clear connection between the abundance of the considered O-bearing methyl and ethyl species. Consequently, the derived lower limit ratio for Met-NCO/Et$\mathrm{NCO}$ may suggest that the production of $\mathrm{CH}_{3} \mathrm{CH}_{2} \mathrm{NCO}$ is not di- 

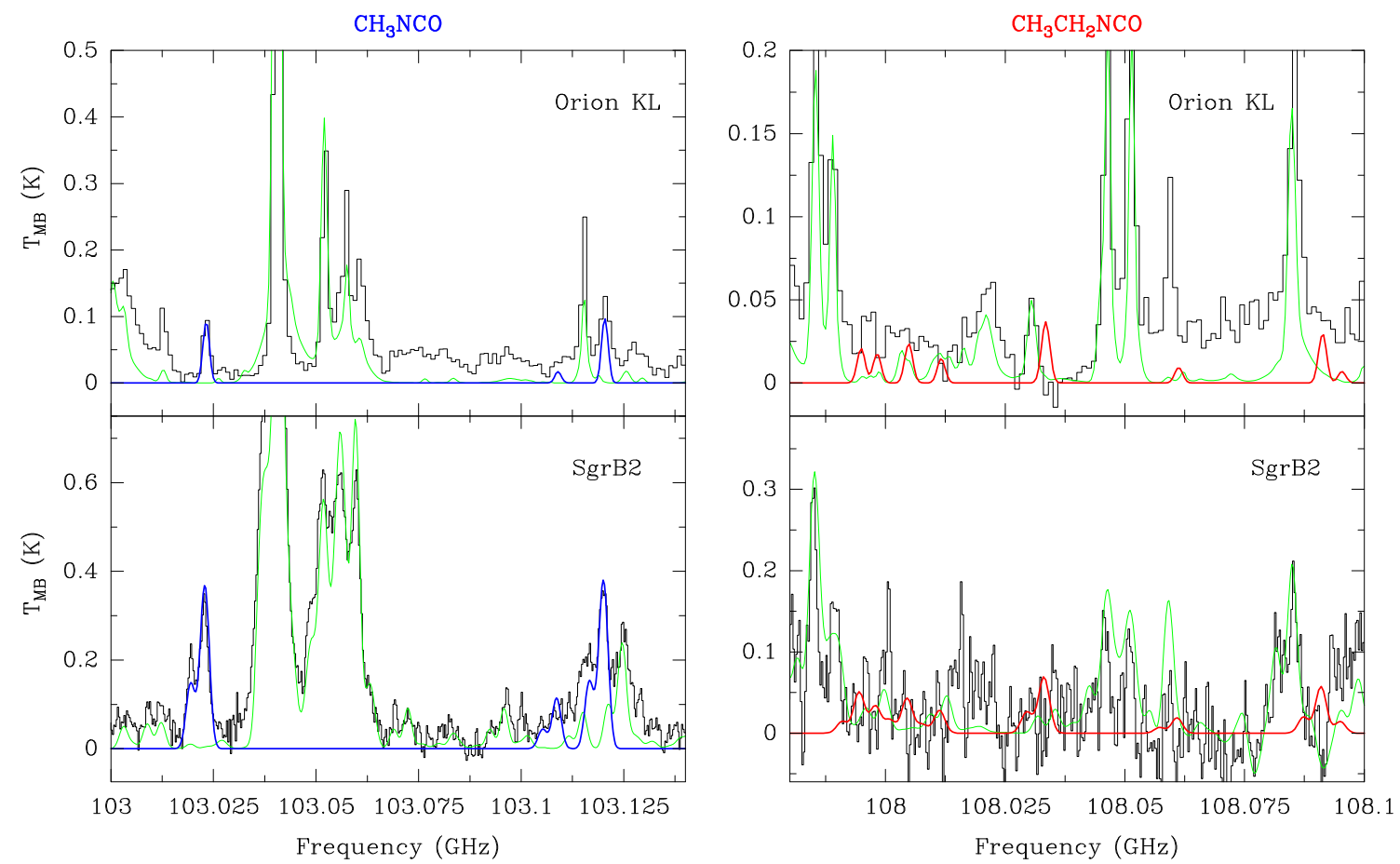

Fig. 3. Selected lines of $\mathrm{CH}_{3} \mathrm{NCO}$ and $\mathrm{CH}_{3} \mathrm{CH}_{2} \mathrm{NCO}$ in Orion $\mathrm{KL}$ and $\mathrm{Sgr} \mathrm{B} 2(\mathrm{~N})$ at $3 \mathrm{~mm}$. Observed data (black histogram spectrum) and total model of the source (green curve) are from Tercero et al. (2010) and Cernicharo et al. (2016) for Orion KL and Belloche et al. (2013) for Sgr B2. Model for $\mathrm{CH}_{3} \mathrm{NCO}$ is given by the blue line (Cernicharo et al. 2016). The synthetic spectrum for $\mathrm{CH}_{3} \mathrm{CH}_{2} \mathrm{NCO}$ corresponding to the upper limit reported in Table 2 is given by the red line. A $v_{\mathrm{LSR}}$ of +9.0 and $+64.0 \mathrm{~km} \mathrm{~s}^{-1}$ is assumed for Orion KL and Sgr B2, respectively.

Table 3. Methyl/ethyl ratios in Orion KL and Sgr B2.

\begin{tabular}{lcc}
\hline \hline Ratio & Orion KL & Sgr B2 \\
\hline Met-NCO/Et-NCO & $\geq 10$ & $\geq 10$ \\
Met-CN/Et-CN & $0.55^{a}$ & $1.35,0.78^{b}$ \\
Met-OH/Et-OH & $45^{c}$ & $21,15^{d}$ \\
Met-OCOH/Et-OCOH & $120^{e}$ & $15,11^{f}$ \\
\hline
\end{tabular}

Notes. ${ }^{(a)}$ López et al. (2014), assuming hot core $\left(v_{\mathrm{LSR}} \simeq 3-5 \mathrm{~km} \mathrm{~s}^{-1}\right)$ and plateau Orion KL components. ${ }^{(b)}$ Belloche et al. (2009), for 63 and 73 $\mathrm{km} \mathrm{s}^{-1}$ velocity components. ${ }^{(c)}$ Tercero et al. (2015), for south hot core $\left(v_{\mathrm{LSR}} \simeq 7-8 \mathrm{~km} \mathrm{~s}^{-1}\right) .{ }^{(d)}$ Belloche et al. $(2009,2013)$, for 63 and $73 \mathrm{~km} \mathrm{~s}^{-1}$ velocity components. ${ }^{(e)}$ Tercero et al. (2015), for the compact ridge of Orion KL. ${ }^{(f)}$ Belloche et al. (2009), for 64 and $74 \mathrm{~km} \mathrm{~s}^{-1}$ velocity components.

rectly linked to the presence of $\mathrm{CH}_{3} \mathrm{NCO}$ in these high-mass starforming regions.

\section{Conclusions}

The rotational spectrum of $\mathrm{CH}_{3} \mathrm{CH}_{2} \mathrm{NCO}$ has been recorded from 80 to $340 \mathrm{GHz}$ and more than 1100 lines were newly assigned for the ground state and three excited vibrational states of the cis conformer. $\mathrm{CH}_{3} \mathrm{CH}_{2} \mathrm{NCO}$ has not been identified above the detection limit of data of the well-know high-mass starforming regions Orion KL and Sgr B2. Finally, it has to be noted that due to the number and complexity of perturbations observed in the rotational spectra of $\mathrm{CH}_{3} \mathrm{CH}_{2} \mathrm{NCO}$, some care has to be taken for predictions and further interstellar searches. Neither interpolations within the range of $J$ and $K_{a}$ values included in this work $(J=13-64$ and $K=0-14)$ nor extrapolations to higher values are recommended and all transitions that are used for interstellar identifications should be checked against experimen- tally measured frequencies collected in the Tables 4 and 5 . Despite this, concerning the data given in the literature up to now, this work has led to a more profound description of the pure rotational spectrum of $\mathrm{CH}_{3} \mathrm{CH}_{2} \mathrm{NCO}$ and constitutes a good base for further searches for it in the ISM.

Acknowledgements. The research leading to these results has received funding from the European Research Council under the European Union's Seventh Framework Programme (FP/2007-2013) / ERC-2013-SyG, Grant Agreement n. 610256 NANOCOSMOS, Ministerio de Ciencia e Innovación (Grants CTQ2013-40717-P, CTQ2016-76393-P, AYA2012-32032, and ConsoliderIngenio 2010 CSD2009-00038 program "ASTROMOL") and Junta de Castilla y León (VA077U16). E.R.A. thanks Ministerio de Ciencia e Innovación for FPI grant (BES-2014-067776). This work was also based on observations carried out with the IRAM 30-meter telescope. IRAM is supported by INSU/CNRS (France), MPG (Germany), and IGN (Spain).

\section{References}

Belloche, A., Garrod, R. T., Müller, H. S. P., et al. 2009, A\&A, 499, 215 Belloche, A., Müller, H. S. P., Menten, K. M., Schilke, P., \& Comito, C. 2013, A\&A, 559, A47

Belloche, A., Meshcheryakov, A. A., Garrod, R. T., et al. 2017, A\&A, 601, A49 Brünken, S., Belloche, A., Martín, S., Verheyen, L., \& Menten, K. M. 2010, A\&A, 516, A109

Cernicharo, J. 2012, in ECLA-2011: Proceedings of the European Conference on Laboratory Astrophysics, Laboratory Astrophysics and Astrochemistry in the Herschel/ALMA Era, 58, 251

Cernicharo, J., Kisiel, Z., Tercero, B., et al. 2016, A\&A, 587, L4

Daly, A., Kolesniková, L., Mata, S., \& Alonso, J. 2014, J. Mol. Spectr., 306, 11 Durig, J. R., Zhou, S. X., Zheng, C., \& Durig, D. T. 2010, J. Mol. Struc., 971, 23 Goesmann, F., Rosenbauer, H., Bredehöft, J. H., et al. 2015, Science, 349 Halfen, D. T., Ilyushin, V. V., \& Ziurys, L. M. 2015, ApJ, 812, L5 Heineking, N., Grabow, J.-U., \& Stahl, W. 1994, Mol. Phys., 81, 1177 Herbst, E., \& van Dishoeck, E. F. 2009, ARA\&A, 47, 427

Kasten, W., Dreizler, H., \& Andresen, U. 1983, J. Mol. Struc., 97, 221 Kisiel, Z., Pszczółkowski, L., Medvedev, I. R., et al. 2005, J. Mol. Spectr., 233, 231, http: //www.ifpan.edu.pl/ kisiel/aabs/aabs.htm\#aabs Ligterink, N. F. W., Coutens, A., Kofman, V., et al. 2017, MNRAS, 469, 2219 López, A., Tercero, B., Kisiel, Z., et al. 2014, A\&A, 572, A44 
Martín-Doménech, R., Rivilla, V. M., Jiménez-Serra, I., et al. 2017, MNRAS, 469, 2230

Pickett, H. M. 1991, J. Mol. Spectr., 148, 371

Sakaizumi, T., Yamada, O., Ushida, K., Ohashi, O., \& Yamaguch, i. I. 1976, Bull. Chem. Soc. Japan, 49, 2908

Snyder, L. E., \& Buhl, D. 1972, ApJ, 177, 619

Sullivan, J. F., Durig, D. T., Durig, J. R., \& Cradock, S. 1987, J. Phys. Chem., 91,1770

Tercero, B., Cernicharo, J., Pardo, J. R., \& Goicoechea, J. R. 2010, A\&A, 517, A96

Tercero, B., Cernicharo, J., López, A., et al. 2015, A\&A, 582, L1 